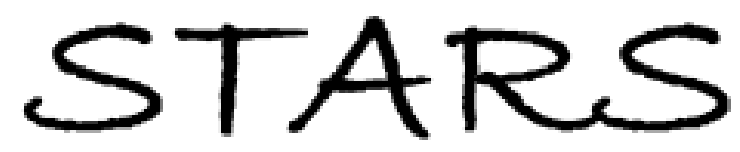

University of Central Florida

STARS

$1-1-2009$

\title{
Development of novel integrated bio/chemical sensor systems using chalcogenide glass materials
}

\author{
Laeticia Petit \\ Nathan Carlie \\ Bodgan Zdyrko \\ Igor Luzinov \\ Kathleen Richardson
}

See next page for additional authors

Find similar works at: https://stars.library.ucf.edu/facultybib2000

University of Central Florida Libraries http://library.ucf.edu

This Article is brought to you for free and open access by the Faculty Bibliography at STARS. It has been accepted for inclusion in Faculty Bibliography 2000s by an authorized administrator of STARS. For more information, please contactSTARS@ucf.edu.

\section{Recommended Citation}

Petit, Laeticia; Carlie, Nathan; Zdyrko, Bodgan; Luzinov, Igor; Richardson, Kathleen; Hu, Juejun; Agarwal, Anu; Kimerling, Lionel; Anderson, Troy; and Richardson, Martin, "Development of novel integrated bio/ chemical sensor systems using chalcogenide glass materials" (2009). Faculty Bibliography 2000s. 2000. https://stars.library.ucf.edu/facultybib2000/2000

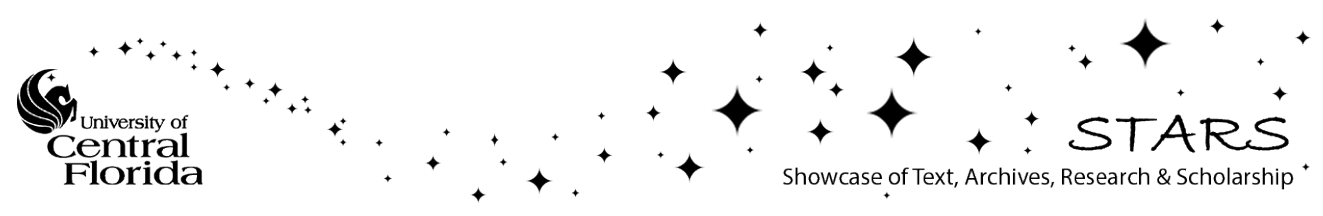




\section{Authors}

Laeticia Petit, Nathan Carlie, Bodgan Zdyrko, Igor Luzinov, Kathleen Richardson, Juejun Hu, Anu Agarwal, Lionel Kimerling, Troy Anderson, and Martin Richardson 


\title{
Development of novel integrated bio/chemical sensor systems using chalcogenide glass materials
}

\section{Laeticia Petit, Nathan Carlie, Bodgan Zdyrko, Igor Luzinov and Kathleen Richardson*}

School of Materials Science and Engineering,

Clemson University,

161 Sirrine Hall, Clemson SC 29634, USA

E-mail: lpetit@clemson.edu

E-mail: ncarlie@clemson.edu

E-mail: bogdanz@clemson.edu

E-mail: Luzinov@clemson.edu

E-mail: richar3@clemson.edu

${ }^{*}$ Corresponding author

\section{Juejun Hu, Anu Agarwal and Lionel Kimerling}

Micro-photonics Center,

Massachusetts Institute of Technology,

77 Mass. Ave., Cambridge, MA 02139, USA

E-mail: hujuejun@mit.edu

E-mail: anu@mit.edu

E-mail: lckim@mit.edu

\author{
Troy Anderson and Martin Richardson \\ College of Optics and Photonics CREOL/FPCE, \\ University of Central Florida, Orlando, \\ 4000 Central Florida Boulevard, FL 32816, USA \\ E-mail: troy@creol.ucf.edu \\ E-mail: mcr@creol.ucf.edu
}

\begin{abstract}
This paper reviews ongoing progress in the design and fabrication of new, on-chip, low loss planar molecular sensors. We report the details of device design, material selection and manufacturing processes used to realise high-index-contrast (HIC), compact micro-disk resonators. These structures have been fabricated in thermally evaporated As- and Ge-based chalcogenide glass films with PDMS (polydimethylsiloxane) micro-fluidic channels using standard UV lithography. Discussed are findings that demonstrate that our novel chalcogenide-based micro-fluidic device can be used as highly sensitive refractive index sensors.
\end{abstract}

Keywords: chalcogenide glass; thin film; waveguide; sensor; resonator; photonic integration; refractometry; absorption spectroscopy; micro-fluidics; lab-on-a-chip. 
Reference to this paper should be made as follows: Petit, L., Carlie, N., Zdyrko, B., Luzinov, I., Richardson, K., Hu, J., Agarwal, A., Kimerling, L., Anderson, T. and Richardson, M. (2009) 'Development of novel integrated bio/chemical sensor systems using chalcogenide glass materials', Int. J. Nanotechnol., Vol. 6, No. 9, pp.799-815.

Biographical notes: Laeticia Petit received her BS and MS Degrees in Physical Chemistry from the University of Pierre et Marie Curie in France and her $\mathrm{PhD}$ in 2002 in Materials Science from the University of Bordeaux in France. She is currently a Research Assistant Professor at Clemson.

Nathan Carlie graduated in 2005 with BS Degree in Chemistry from the University of Central Florida, and is currently a PhD candidate in the School of Materials Science and Engineering at Clemson University.

Bogdan Zdyrko received his $\mathrm{PhD}$ in Materials Science and Engineering from Clemson University, Clemson, USA. He worked as a Post-Doctoral research Associate at the Department of Polymer Science and Engineering, University of Massachusetts, Amherst, USA.

Igor Luzinov is an Associate Professor of Polymer Science Clemson University. He received a $\mathrm{PhD}$ Degree in Polymer Chemistry in 1990 from Lviv Polytechnic National University (Ukraine). He served as a Senior Research Scientist at Physical Chemistry Institute (National Academy of Science of Ukraine), NATO Research Fellow at University of Liege, Belgium and Postdoctoral Research Associate at Iowa State and Western Michigan Universities.

Kathleen Richardson is Director of the School of Materials Science and Engineering at Clemson and leads the Glass Processing and Characterisation Laboratory (GPCL). She received her PhD in 1992 from the NYS College of Ceramics at Alfred University and is a Fellow of the American Ceramic Society, SPIE and Society of Glass Technology.

Juejun $\mathrm{Hu}$ received the BS Degree in Materials Science from Tsinghua University, Beijing, China, in 2004. He is currently working toward the $\mathrm{PhD}$ Degree in the Microphotonics Center at MIT.

Anu Agarwal is a Research Associate in the Microphotonics Center at MIT. She develops integrated Si-CMOS compatible materials and devices, including sensors and imagers. She spent three years at Clarendon Photonics, developing a novel optical filter. Her Doctoral Degree in Electrical Engineering is from Boston University.

Lionel Kimerling is the Thomas Lord Professor of Materials Science and Engineering and Director of the Microphotonics Center at MIT. $\mathrm{He}$ works on the fundamental science of the design and fabrication of microphotonic materials and devices. He is the Creator and Co-Director of the Communications Technology Roadmap which provides technology development targets for the long-term evolution of photonic component integration in the optical communications industry. He received his BS and $\mathrm{PhD}$ Degrees from MIT in 1965 and 1969 respectively.

Troy Anderson received the BS Degree in electrical engineering from the University of Nebraska at Lincoln in 2004 and the MS Degree in optics from the University of Central Florida in 2007. He is currently working towards 
a PhD Degree in Optics at the University of Central Florida in the field of femtosecond laser interactions with matter.

\begin{abstract}
Martin Richardson is the Northrup Grumman Professor of X-ray Photonics and Director of the Townes Laser Institute in the College of Optics and Photonics at the University of Central Florida. He currently leads the Laser Plasma Laboratory where research in fs laser development and materials processing of glasses and other materials is ongoing.
\end{abstract}

\title{
1 Introduction
}

Recent advances in micro-fabrication technology have enabled a paradigm shift in the field of chemical and biological detection. Miniaturised sensing devices can now be created that outperform and possibly replace their conventional bulky counterparts. These devices build on the knowledge base and technologies developed in micro-photonics, micro-fluidics, and micro-electronics and have given rise to sensing/characterisation platforms commonly referred to as a 'sensor-on-a-chip' or 'lab-on-a-chip' [1]. The competitive advantages of such miniaturised sensing devices are three-fold. Firstly, the Moore's law paradigm that is driving the rapid strides of micro-electronics can be applied to revolutionise bio-/chemical detection to leverage mature silicon CMOS manufacturing technology to achieve high volume production and thus very low cost, as well as scalable performance improvement [2]. Secondly, a small device footprint opens up avenues of new device and system applications such as remote sensor network deployment. These networks for example are impossible (cost prohibitive) with a conventional design. Finally, integration of different functional components onto a planar platform enables multi-modal detection and significantly enhanced intelligence capabilities.

Our team's research strategy aims to address critical challenges for realising such a sensor-on-a-chip concept, including development of specialty glass materials and surface coatings using engineered glasses with defined (CMOS) process response and behaviour, compositionally-tailored polymers that meet demanding response and stability performance requirements of advanced gas or liquid phase sensor systems, and novel device geometries and platforms capable of providing both high sensitivity and specificity, over large areas. Recent efforts in our group have focused on the development of a novel, integrated sensor system with low loss, enhanced sensitivity and specificity suitable for use in advanced detection in chemical-biological warfare and other intelligent sensing applications [3-8]. These efforts have resulted in the development of Si-CMOS compatible processing procedures and demonstration of viability of manufacturing uniformity to large areas ( $>6$ " diameter wafers) needed to evaluate scalability and economics in fabricating and integrating optical structures and devices for use in chip-based chemical and/or biological sensors. The novelty of the resulting sensor design is three-fold:

1 Novel high refractive index chalcogenide glass $(\mathrm{ChG})$ materials suitable for multi-spectral chemical and biological sensing have been developed which allow extension of the operating wavelength range for devices from the UV to visible to far infrared; these glasses enable use of a low temperature process to fabricate an 
ultra-high-Q optical resonant cavity with an atomically smooth surface. Although being widely used as phase change materials for optical disks and non-volatile random access memories, $\mathrm{ChG}$ films are also good candidates for planar integrated nonlinear optical devices due to their high nonlinearities and low linear and nonlinear loss, their fabrication and structural flexibility, wide range of optical and electrical properties, large capacity for doping, and tailorable photosensitivity [9-12]. Several micro-photonics devices such as gratings, optical storage units, holographic recordings, optical amplifiers and lasers that utilise these unique properties of chalcogenides, have already been demonstrated [13]. ChGs exhibit a broad optical transparency window stretching from the visible to the far-infrared, allowing the realisation of multi-modal optical detection in the same material platform, a significant advantage for device processing and integration [14]. In addition, the high refractive index of chalcogenide glasses enables compact photonic integration essential for array-format high-throughput analysis; most importantly, their almost infinite capability of compositional alloying enables application-specific tailoring of glass properties such as thermo-optic coefficient, optical response (damage resistance, photo-induced nonlinear response) and chemical compatibility with sensing molecules in different environments.

2 In conventional bulk optical sensor design, high sensitivity is achieved via increased optical path length not amenable to device miniaturisation and scaling [15]; in contrast, our approach relies on planar optical resonators to enhance the device sensitivity utilising strong photon-molecule interactions in a resonant cavity while dramatically reducing the sensor footprint. Further, optical resonators offer a versatile device platform suitable for cavity-enhanced absorption and fluorescence spectroscopy as well as label-free refractometric sensing. Finally, compared to fibre-based counterparts, planar ChG-based resonator devices are more mechanically robust and amenable to lower cost, larger-scale integration with other on-chip photonic and electronic devices, enabling a full spectrum of optical signal read-out and processing functions.

3 The sensor device fabrication strategies developed and optimised in this effort employs CMOS compatible processing technology suitable for mass production and further scalable performance improvement. Such scalability reduces per sensor costs allowing multi-sensor arrays tailorable to single or multi-species detection to be deployed for large sampling area evaluation. No other current technology offers such possibility.

In this paper, we review the choice of the materials used for the device fabrication. Secondly we discuss the use of a Si CMOS-compatible fabrication technique for the production of a chalcogenide glass-based sensor monolithically integrated on a silicon platform. We demonstrate the ability to fabricate

- single-mode waveguides with core sizes down to the sub-micron range possessing reduced sidewall roughness using a lift-off technique that has broad applicability to a diverse range of non-silica glass compositions and also

- chalcogenide glass racetrack and micro-disk resonators, the key elements for biochemical sensing. 
Lastly, we describe how these devices can be used as highly sensitive absorption spectroscopy and refractometry sensors.

\section{Experimental}

\subsection{Glass preparation and film deposition}

The synthesis of the As- and Ge-based glasses used in this study is explained in detail, elsewhere [16,17]. All glasses are prepared from high purity elements (As Alfa 99.999\%, Ge Aldrich 99.999\%, Sb Alpha 99.9\% and S Cerac 99.999\%) with no further purification. Starting materials are weighed and batched into quartz ampoules inside a nitrogen-purged glove box and sealed under vacuum using a gas-oxygen torch. Prior to sealing and melting, the ampoule and batch are pre-heated at $100^{\circ} \mathrm{C}$ for $4 \mathrm{~h}$ to remove surface moisture from the quartz ampoule and the batch raw materials. The ampoule is then sealed and heated for $24 \mathrm{~h}$ at between $800^{\circ} \mathrm{C}$ and $975^{\circ} \mathrm{C}$, depending on the glass composition. A rocking furnace is used to rock the ampoule during melting to increase melt homogeneity. Once homogenised, the melt-containing ampoule is air-quenched to room temperature. To avoid fracture of the tube and glass ingot, ampoules are subsequently returned to the furnace for annealing for $15 \mathrm{~h}$ at $40^{\circ} \mathrm{C}$ below the respective glass transition temperature, $T_{g}$, of the glass. Glass samples are then cut, optically polished and inspected visually. These bulk glasses are targets for subsequent film deposition. Glass composition has been verified using elemental dispersive spectroscopy (EDS) and found to be identical to the initial batch composition. No loss of sulphur has been observed within the accuracy of the measurement $( \pm 2$ at $\%)$. $\mathrm{X}$-ray diffraction (XRD) has been carried out to confirm the amorphous nature of our samples.

As- and Ge-based thin films on oxide-coated $\mathrm{Si}$ wafers (6" Si wafers with $3 \mu \mathrm{m}$ thermal oxide, Silicon Quest International) and glass microscope slides were prepared via thermal evaporation and/or pulsed laser deposition methods using bulk glass materials as targets. A complete description of the techniques for the As-based film deposition [18] and that for Ge-based glasses [6] can be found elsewhere. Most recently, deposition of the Ge-based films prepared using thermal evaporation were made at a base pressure of $2 \times 10^{-7}$ Torr using a alumina-coated tantalum baffled source in a custom-designed thermal evaporator (model 112 Evaporator-Sputter Station from PVD Products Inc.), and the deposition rate is stabilised at $18 \AA / \mathrm{s}$ [6]. A thermostat stage is also employed to maintain the substrate temperature at $25^{\circ} \mathrm{C}$ throughout the deposition process. Pulsed laser deposition (PLD) film formation is carried out using a mode-locked $\mathrm{Nd}: \mathrm{YVO}_{4}$ laser operating at third harmonic $(355 \mathrm{~nm})$ and delivering $\sim 5 \mathrm{~W}$ to the target surface [19]. The repetition rate is $28 \mathrm{MHz}$; pulse duration $12 \mathrm{ps}$; focal spot size $\sim 15 \mu \mathrm{m}$; maximum incident intensity $\sim 10^{10} \mathrm{~W} / \mathrm{cm}^{2}$ with the fluence $0.1 \mathrm{~J} / \mathrm{cm}^{2}$. The laser beam is scanned using $x-y$ scanning mirrors in a constant velocity $2 \mathrm{~m} / \mathrm{s}$ spiral over the area of approx. $2.5 \mathrm{~cm}^{2}$ to prevent drilling craters into the target surface. The substrate is located at $\sim 160 \mathrm{~mm}$ from the target and rotated to provide a more homogeneous film thickness over the substrate area. The base pressure in the deposition chamber is $5.0 \times 10^{-7}$ Torr. No evidence of crystallisation or phase separation has been observed in either the As- or Ge-based films. A detailed comparison of glassy films prepared by these two techniques is forthcoming [20]. 


\subsection{Characterisation of sensor/resonator}

The morphology of as-fabricated waveguides has been characterised using a JEOL 6320FV field-emission high-resolution SEM. A Digital Instruments Nanoscope IIIa Atomic Force Microscope (AFM) has been used to measure the roughness of the as-patterned waveguides. Measurement scans have been performed parallel to the propagation direction of the waveguides using the tapping mode. Waveguide loss measurements have been performed on a Newport AutoAlign workstation. Lens-tip fibres have been used to couple laser light into and out of the waveguides. Highly reproducible coupling between waveguides and fibres can be achieved via an automatic alignment system. Waveguide loss has been determined via a standard cutback method using paper-clip waveguide patterns.

\section{Glass material down-selection}

To design and fabricate the planar molecular sensor, the candidate infrared (IR) glass for the optical resonator structure must possess the following attributes:

- The glass needs to be stable against devitrification as indicated by a difference between crystallisation $\left(T_{x}\right)$ and glass transition $\left(T_{g}\right)$ temperatures larger than $90^{\circ} \mathrm{C}$. Moreover, $T_{g}$ needs to be high enough to remain stable during processing of surface coatings and to minimise aging for refractometry sensing

- As most biological and chemical agents have their identification signatures in the IR range, the glass candidate needs to have low absorption (including electronic and bond vibrational absorption) at the operation wavelengths (for instance, mid- and far-infrared wavebands for absorption spectroscopic sensing, near-infrared water transparency window for refractometric sensing and fluorophore excitation wavelength for fluorescence spectroscopy) for sensitivity improvement, which in turns dictates the application-specific glass phonon energy and optical band gap.

- The glass also needs to possess a high linear refractive index to allow compact device design and to minimise substrate leakage loss.

- The process compatibility with large-area film deposition techniques (e.g., evaporation or sputtering) is critical for device fabrication; also of importance is the thermal/chemical stability during all steps in the lithographic process.

- Chemical stability and material durability in the sensing environment, and biological compatibility for biosensor applications (which could also be possibly achieved through appropriate surface coatings using specific polymers) also define further material requirements.

- The optical band gap of the film needs to be low enough to induce effective 2-photon absorption when irradiated with near-IR (NIR) $[\lambda=800 \mathrm{~nm}]$ femtosecond laser for three dimensional (3D) waveguide writing. 
- The compositionally-tailored photo-sensitivity should be suitable for NIR fs laser structuring with desired photo-induced refractive index change (positive) with no photo-induced crystallisation and limited aging effects.

Several years ago researchers within our team demonstrated that As-based glasses

- $\quad$ are very stable against crystallisation

- can be deposited into films using thermal evaporation

- $\quad$ when exposed to near-IR $(800 \mathrm{~nm})$ femtosecond laser exhibit an increase of refractive index $[16,21]$.

A systematic study [16] examined the compositional dependences of such attributes including linear and nonlinear indices and absorption properties and the influence on photo-induced structural changes, on these properties. While versatile from the standpoint of photosensitivity and high linear and nonlinear indices, many binary and ternary ChG glasses in the As-Se, As-S and As-S-Se systems have low glass transition temperatures $\left(\sim 200^{\circ} \mathrm{C}\right)$, and also exhibit mediocre long-term stability. These limitations are driven by the glass' constituent elements, which while suitable for long wave optical transmission within the two colour IR bands, possess thermal-mechanical robustness far less than oxide counterparts. Hence, versatility in the IR (bandwidth to allow transmission and detection of IR resonant species) is at the expense of good processability and long-term performance. To offset this limitation, we have extended the stability of glasses by enhancing the coordination of the glass network, making glasses with higher thermal stability amenable for use in CMOS processing and high stability sensor devices. For this reason, we have included in our study Ge-based glasses which not only bring the rigidity of a four-coordinated network former, but also serves to eliminate the use of As which is undesirable in CMOS manufacturing environments. Ge-containing glass is known to exhibit higher melting and glass transition temperatures than the As-based glasses [22]. We have demonstrated that $\mathrm{Ge}_{23} \mathrm{Sb}_{7} \mathrm{~S}_{70}$ glass can be reproducibly deposited into thin films using thermal evaporation [20]. While suitable for CMOS lithographic processing, this glass cannot be used for direct write waveguides as we have shown [23]. Anderson et al. showed that near-IR fs laser irradiation at both $\mathrm{kHz}$ and $\mathrm{MHz}$ repetition rates leads to a decrease of the refractive index attributed to a modification of bonding between the glass' units that form the well organised 3D network. This resulting network modification is in agreement with [24]. As a result, we can use either Ge- or As- based glasses depending on the type of structuring/device fabrication we desire. In our current study, we have primarily utilised As- and Ge-based glasses for fabrication of waveguides, rings and resonators fabricated using lithographic processing; for the 3D waveguide writing using direct laser, we have only focused our effort on the As-based glasses system. In this paper, we summarise our most recent results on devices fabricated using lithographic processing.

\section{Device fabrication}

The novel sensor device discussed above is comprised of three parts: a micro-fluidic flow system for analyte transport, an optical sensing unit for generating signals modulated by 
the presence of targeted chemical or biological species, and peripheral electronics for signal read-out and processing. At the core of the device, the optical sensing unit consists of several sequential components: a light source to emit the probing light, planar waveguides to couple the light into the resonator, polymer coated on-chip optical resonators where strong light-chemical interactions take place, and finally photo-detectors to receive the signal through waveguides that couple the light. In this section, we describe the device fabrication steps from the film deposition through the planar waveguide fabrication.

\subsection{Film deposition}

There are several previously reported methods for preparation of $\mathrm{ChG}$ thin films including, chemical reaction from the vapour phase (CVD) [25], thermal evaporation (TE) from bulk target materials [13], sol-gel [26], RF magnetron sputtering [27], and pulsed laser deposition (PLD) [19,28,29]. As the physical process and kinetics of these techniques are quite different, these deposition techniques can often produce films with differing optical and thermal properties.

Glassy thin films with the composition $\mathrm{Ge}_{23} \mathrm{Sb}_{7} \mathrm{~S}_{70}$ deposited using the thermal evaporation and the pulsed laser deposition techniques have been found to have similar thermal and optical properties [20]. Using micro-Raman spectroscopy, we showed that both as-deposited films have similar network structures, but both differ from that of the corresponding parent bulk glass. This we attributed to the fact that the film deposition process impacts the film density, specifically the molecular organisation of constituents during film formation; the TE and PLD processes yield glassy films containing homopolar Ge-Ge bonds, with a decrease in homopolar S-S bonds leading to an increase in $\mathrm{GeS}_{4 / 2}$ units. These observations are in agreement with [28,30,31]. A comparison of the structural entities associated with each deposition process showed that the TE film yields a lower number of S-S bonds and a slightly higher number of $\mathrm{SbS}_{3 / 2}$ units compared to that of the PLD film. These structural features were found to be consistent with the observation of a more dense network structure which would result in a higher refractive index, with the glass' optical band gap position shifted to longer wavelength.

In the current study, the thermal evaporation technique has been used for the deposition of the films with the composition $\mathrm{Ge}_{17} \mathrm{Sb}_{12} \mathrm{~S}_{71}$ and of the As-based films with the composition $\mathrm{As}_{42} \mathrm{~S}_{58}$ and $\mathrm{As}_{2} \mathrm{~S}_{3}$. TE was chosen as the method of choice given its

1 low-cost, large-area uniformity (vs. PLD) and simplicity

2 high-throughput (compared to sputtering)

3 minimised step coverage which proves excellent for subsequent lift-off (vs. CVD).

Thermal evaporation is often a non-congruent vapourisation process which can lead to off-stoichiometry films; in this case it is crucial to modify the starting bulk composition to compensate for this change to yield films and structures with desired target refractive index.

Using a SEM, the As-based films did not present any obvious signs of local compositional variation or phase separation, as evident from uniform elemental maps made with energy dispersive spectroscopy (EDS) across multiple $1 \mathrm{~mm}^{2}$ areas of sample surface. We demonstrated that the thermal evaporation process results in As-based films 
with similar composition and thermal properties as compared to those of the parent bulk glasses [20]. However, as observed for some Ge-films $\left(\mathrm{Ge}_{23} \mathrm{Sb}_{7} \mathrm{~S}_{70}\right)$ via Raman spectroscopy, the structure of the As-based films, contains a significantly higher number of $\mathrm{As}_{4} \mathrm{~S}_{4}$ molecular units as well as some $\mathrm{As}_{4} \mathrm{~S}_{3}$ units. We believe these species, found to be only present in films, result from the different thermal history (quench rate) induced during the film deposition process [20].

The study of the structure of the as-deposited As-based and Ge-based films confirms that the deposition of glasses into thin films can result in variation of the material properties which may affect device performance. Knowledge of the effects of such processing conditions on relevant glass properties, such as refractive index, structure and glass transition temperature, and definition of means to correct or compensate for them, is crucial for the choice of the glasses used for the device design and fabrication.

\subsection{Waveguide and resonator fabrication}

The waveguide, micro-ring and micro-resonator structures have been fabricated using the lift-off process. As in a standard lift-off process, a photoresist pattern is first formed on a substrate, in the present study, a blank oxide-coated $\mathrm{Si}$ wafer. ChG film is then thermally evaporated onto the wafer patterned with photoresist, and sonicated in solvent (usually acetone) to dissolve the photoresist layer beneath the undesired parts of the film, thus lifting it off. Only glass deposited onto areas not covered by photoresist is retained, and thus a chalcogenide pattern reversed to that of the photoresist is defined. The patterned wafer is then rinsed in methanol and isopropanol to clean the surface. To fabricate rib waveguides, a second film deposition is made sequentially on a lift-off patterned film.

In our process, the starting substrates are coated (with a $3 \mu \mathrm{m}$-thick thermal oxide) 6" Si wafers. Commercially available negative resist NR9-1000PY (Futurrex Inc.) is used due to its negative-sloping sidewall profile and superior pattern resolution. The resist is spin-coated onto substrates on a manual photoresist coater (Model 5110, Solitec Inc.). UV exposure is carried out using a Nikon NSR-2005i9 i-line wafer stepper (minimum linewidth $500 \mathrm{~nm}$ ). Resist pattern development and subsequent baking are both completed on an SSI 150 automatic photoresist coater/developer track. The entire photolithography process is performed in a class-10 CMOS clean room. To prevent surface oxidation, a $3 \mu \mathrm{m}$ thick layer of SU8 polymer is spin-coated to serve as a top cladding after patterning and the devices are subsequently annealed at $140^{\circ} \mathrm{C}$ for $3 \mathrm{~h}$ to stabilise the glass structure, whereas no SU8 coatings are applied on the waveguides and resonators. Figure 1(a) shows a cross-sectional SEM micrograph of a strip $\mathrm{Ge}_{23} \mathrm{Sb}_{7} \mathrm{~S}_{70}$ waveguide before photoresist lift-off and (b) an AFM scan of a $2 \mu \mathrm{m}$ by $2 \mu \mathrm{m}$ square area showing the surface morphology of a waveguide with a width of $750 \mathrm{~nm}$, respectively.

The AFM measurements yield an average sidewall line RMS roughness value of $(11 \pm 2) \mathrm{nm}$ for as-fabricated waveguides and $(1.6 \pm 0.3) \mathrm{nm}$ for the strip waveguide's top surface. In comparison, plasma etched $\mathrm{Ge}_{23} \mathrm{Sb}_{7} \mathrm{~S}_{70}$ waveguide sidewalls exhibit RMS roughness values typically ranging from $20-150 \mathrm{~nm}$ depending on the etching parameters [7]. The relatively low sidewall roughness in waveguides fabricated through lift-off can be attributed to the fact that the sidewall is defined during a deposition process rather than etching. In the lift-off process, we believe the major source of sidewall roughness originates only from edge roughness of photoresist patterns. 
Figure 1 (a) Cross-sectional SEM micro-graph of a sub-micron $\mathrm{Ge}_{23} \mathrm{Sb}_{7} \mathrm{~S}_{70}$ strip waveguide before photoresist lift-off and (b) AFM morphological scan of a $\mathrm{Ge}_{23} \mathrm{Sb}_{7} \mathrm{~S}_{70}$ strip waveguide fabricated via the lift-off method (see online version for colours)

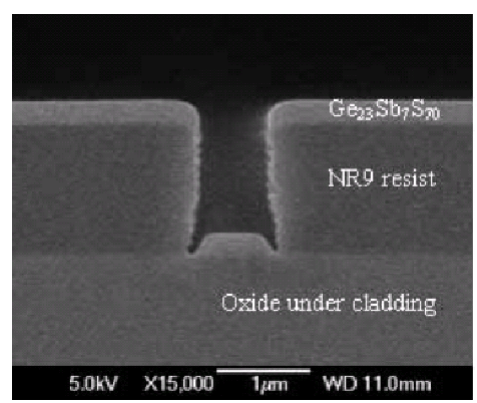

(a)

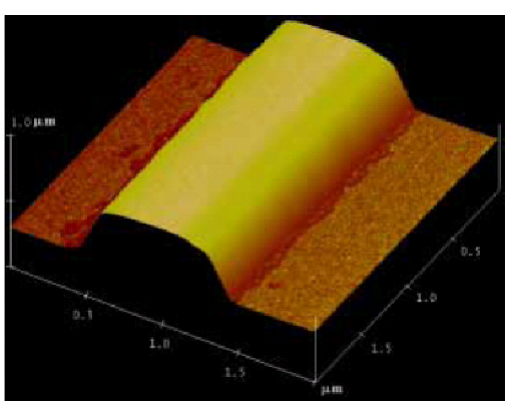

(b)

Figure 2(a) shows the top view of a fabricated $\mathrm{As}_{2} \mathrm{~S}_{3}$ racetrack resonator and (b) a $20 \mu \mathrm{m}$-radius $\mathrm{As}_{2} \mathrm{~S}_{3}$ micro-disk resonator with a bus waveguide in a 'pulley-type' coupling configuration. Compared to a conventional micro-disk/micro-ring coupler, the pulley coupler design increases the coupling length leading to stronger coupling. In order to achieve the same coupling strength as a conventional coupler design, a wider gap between a resonator and a bus waveguide is possible when a pulley coupler is employed. With such performance constraints reduced, lower cost lithography techniques can be used for fabrication.

Figure 2 Top view optical micro-graph of (left) a racetrack $\mathrm{As}_{2} \mathrm{~S}_{3}$ resonator and (right) an $\mathrm{As}_{2} \mathrm{~S}_{3}$ micro-disk resonator with a pulley coupler configuration (see online version for colours)
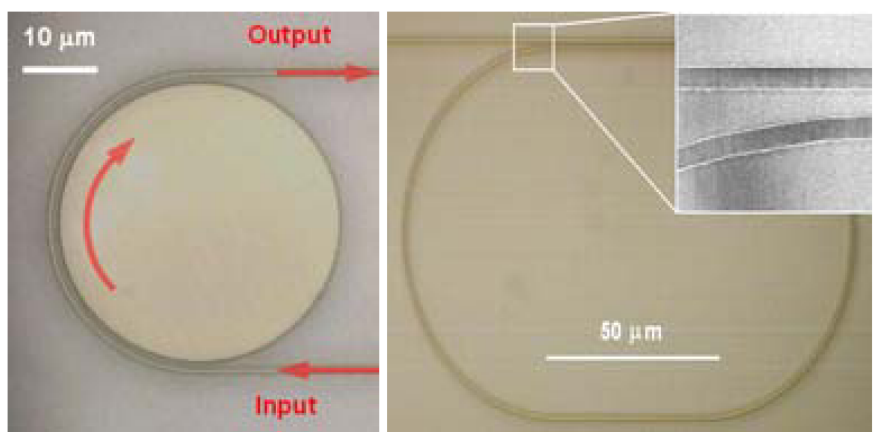

\section{Device testing}

\subsection{Waveguide loss measurement}

Transmission loss measurements made at $1550 \mathrm{~nm}$ for strip and rib $\mathrm{Ge}_{23} \mathrm{Sb}_{7} \mathrm{~S}_{70}$ waveguides of different widths and cross-sectional geometry are reported in Table 1. 
Table 1 Measured optical transmission losses and calculated modal parameters of $\mathrm{Ge}_{23} \mathrm{Sb}_{7} \mathrm{~S}_{70}$ waveguides at $1550 \mathrm{~nm}$ wavelength

\begin{tabular}{lcccccc}
\hline \multirow{2}{*}{$\begin{array}{l}\text { Waveguide } \\
\text { width }(\mathrm{w})\end{array}$} & \multicolumn{2}{c}{$0.75 \mu m$ (strip) } & \multicolumn{2}{c}{$1.2 \mu m$ (strip) } & $1.6 \mu m$ (strip) & $1.2 \mu m$ (rib) \\
\cline { 2 - 7 } & $T M$ & $T E$ & $T M$ & $T E$ & $T M$ & $T E$ \\
\hline $\begin{array}{l}\text { Transmission } \\
\text { loss }(\mathrm{dB} / \mathrm{cm})\end{array}$ & $3.9 \pm 0.4$ & $6.4 \pm 0.8$ & $3.5 \pm 0.3$ & $5.2 \pm 0.5$ & $2.3 \pm 0.4$ & $<0.5$ \\
$\begin{array}{l}\text { Number of } \\
\text { modes } \\
\text { supported }\end{array}$ & 1 & 1 & 1 & 2 & 1 & 1 \\
\hline
\end{tabular}

It can clearly be seen that the Transverse-Magnetic (TM) mode exhibits lower transmission loss than the Transverse-Electric (TE) mode in strip waveguides with the same width. Moreover, the transmission losses for both modes decrease as the strip waveguides become wider. The loss dependence on width is more significant for TE mode than for TM mode. The rib waveguides show very low loss for both TE and TM modes due to less mode interaction with sidewall roughness in the rib waveguide geometry. Statistical analysis reveals that these waveguides have an average loss of $(2.3 \pm 0.4) \mathrm{dB} / \mathrm{cm}$ at $1550 \mathrm{~nm}$ wavelength, larger than those reported by Madden et al. [32] and Choi et al. [33] who measured 0.05 and $0.3 \mathrm{~dB} / \mathrm{cm}$ optical losses at $1550 \mathrm{~nm}$ in $\mathrm{As}_{2} \mathrm{~S}_{3}$ planar rib waveguides with length up to $22.5 \mathrm{~cm}$ and in $\mathrm{Ge}_{33} \mathrm{As}_{12} \mathrm{Se}_{55}$ rib waveguides of 3,4 and $5 \mu \mathrm{m}$ wide, respectively. The standard deviation of $0.4 \mathrm{~dB} / \mathrm{cm}$ is low enough to confirm the excellent processing uniformity of our lift-off technique, indicating that the lift-off is intrinsically a wafer-scale processing technique suitable for scale up for mass production.

Micro-disks with the composition $\mathrm{As}_{2} \mathrm{~S}_{3}$ and $\mathrm{Ge}_{17} \mathrm{Sb}_{12} \mathrm{~S}_{71}$, a radius of $20 \mu \mathrm{m}$ and varied waveguide-resonator gap separation from $500 \mathrm{~nm}$ to $1200 \mathrm{~nm}$ have been also tested. Table 2 summarises the optical properties of the resonators.

Table 2 Coupled cavity Q and free spectral range (FSR) of the micro-disk resonators at $1550 \mathrm{~nm}$

\begin{tabular}{lccccc}
\hline & \multicolumn{2}{c}{ Cavity $Q( \pm 10 \%)$} & & \multicolumn{2}{c}{$F S R(\mathrm{~nm})$} \\
\cline { 2 - 3 } \cline { 5 - 6 } Bulk composition & $T M$ & $T E$ & & $T M$ & $T E$ \\
\hline $\mathrm{As}_{2} \mathrm{~S}_{3}$ & 210,000 & 150,000 & & 7.62 & 7.82 \\
$\mathrm{Ge}_{17} \mathrm{Sb}_{12} \mathrm{~S}_{71}$ (in air) & 110,000 & 100,000 & & 8.35 & 8.92 \\
$\mathrm{Ge}_{17} \mathrm{Sb}_{12} \mathrm{~S}_{71}$ (in water) & 20,000 & & & 8.38 & \\
\hline
\end{tabular}

The higher Q factor of TM polarisation suggests that bending loss is insignificant in the micro-disk, and thus it is possible to achieve an even smaller cavity mode volume without suffering excess radiative loss. The measured transmission spectra of the $\mathrm{As}_{2} \mathrm{~S}_{3}$ micro-disk with a gap separation of $800 \mathrm{~nm}$ between bus waveguide and micro-disk is shown in Figure 3. The transmission spectra feature a set of resonant peaks evenly spaced by a well-defined free spectral range, indicative of single-mode resonator operation. The micro-disk operates near critical coupling regime for both TE and TM polarisations around $1550 \mathrm{~nm}$ wavelength, an important advantage for applications in the telecommunication bands. 
Figure 3 (left) Measured transmission spectra of a $20 \mu$ m-radius $\mathrm{As}_{2} \mathrm{~S}_{3}$ microdisk resonator; (right) a TM-polarisation transmission spectrum averaged over 32 wavelength sweeping scans near a resonant peak: the black dots are experimental data points and the red curve is the Lorentzian peak fitted in linear scale (see online version for colours)
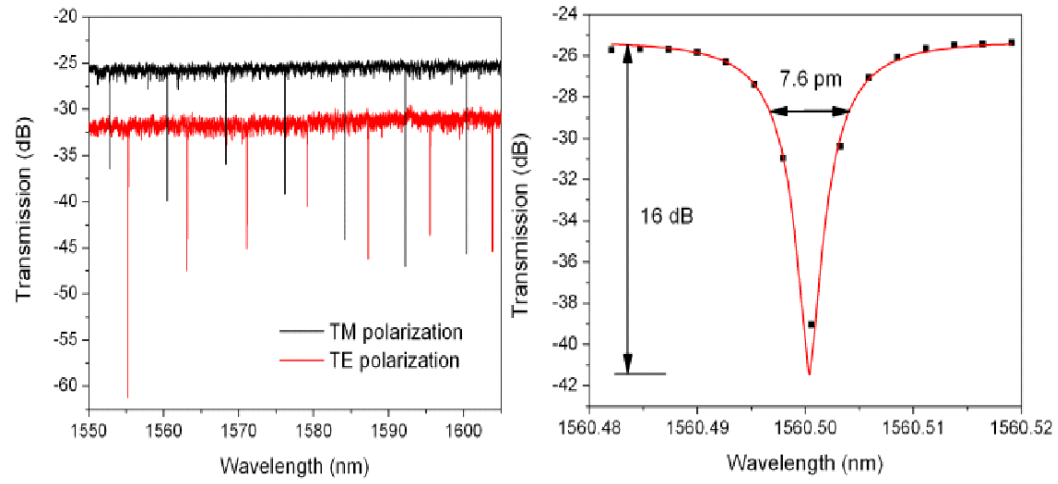

\subsection{Sensor performance testing}

We have tested two types of sensing mechanisms using the same material platform: waveguide evanescent absorption spectroscopy and resonator refractometry. In both applications, replica-molded PDMS (polydimethylsiloxane) micro-fluidic channels are bonded onto the $\mathrm{Ge}_{23} \mathrm{Sb}_{7} \mathrm{~S}_{70}$ glass waveguides or resonators for fluid analyte transport. The details of each design is discussed.

\subsubsection{Testing of the waveguide evanescent absorption sensor performance}

Waveguide evanescent absorption sensor performance is tested by monitoring the optical output while injecting a solution of $\mathrm{N}$-methylaniline in carbon tetrachloride into the micro-fluidic channel. The N-H bond in N-methylaniline is known to exhibit an absorption peak near $1500 \mathrm{~nm}$, which is used as the characteristic fingerprint for chemical identification in our test [34]. The absorption (in $\mathrm{dB}$ ), $\alpha L$, induced by $\mathrm{N}$-methylaniline in our micro-fluidic channel is calculated by taking the ratio of light intensity transmitted through a micro-fluidic channel filled with pure carbon tetrachloride $\left(I_{\text {solvent }}\right)$ and through a channel filled with $\mathrm{N}$-methylaniline solution in carbon tetrachloride ( $\left.I_{\text {analyte }}\right)$ ( 0.33 , volumeric concentration) using:

$$
\alpha L=10 \log _{10} \frac{I_{\text {solvent }}}{I_{\text {analyte }}}
$$

where $L$ is the length of the waveguide immersed in analyte solution (cm). The same waveguide is used throughout the experiment to eliminate any uniformity issue associated with waveguide intrinsic loss. The resultant absorption spectrum shown in Figure 4(a) exhibits a well-defined absorption peak at $1496 \mathrm{~nm}$, which is in excellent agreement with a traditional absorption measurement carried out on a Cary 5E UV-Vis-NIR dual-beam spectrophotometer as seen in Figure 4(b). 
Figure 4 (a) Absorption spectrum showing the N-H bond absorption at $1496 \mathrm{~nm}$ wavelength in N-methylaniline measured with our waveguide evanescent sensor. (b) Transmission spectra of pure $\mathrm{N}$-methylaniline and carbon tetrachloride $\left(\mathrm{CCl}_{4}\right)$

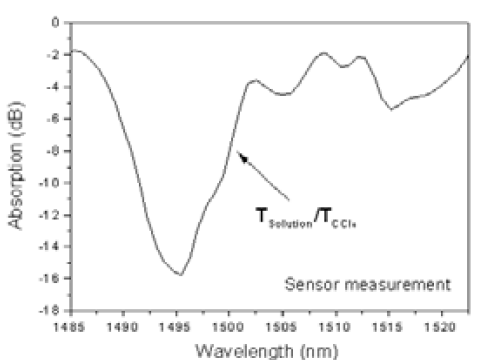

(a)

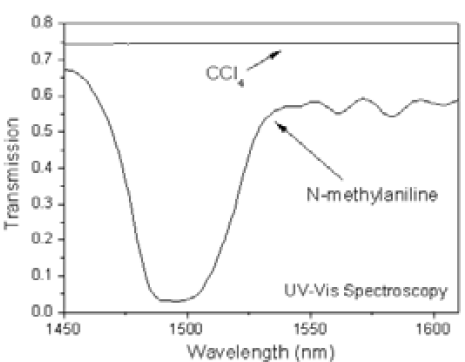

(b)

Since carbon tetrachloride has no absorption band and is transparent in the investigated spectral range, this peak is unambiguously assigned to $\mathrm{N}-\mathrm{H}$ bond vibrational absorption. The peak absorption in $\mathrm{dB}$ at $1496 \mathrm{~nm}$ is measured for different concentrations of $\mathrm{N}$-methylaniline solution in carbon tetrachloride and the result is shown in Figure 5. The excellent linear fit suggests that the sensor exhibits linear response when varying analyte concentrations in the range investigated.

Figure 5 Peak absorption of N-methylaniline solution in carbon tetrachloride measured as a function of $\mathrm{N}$-methylaniline volume concentration

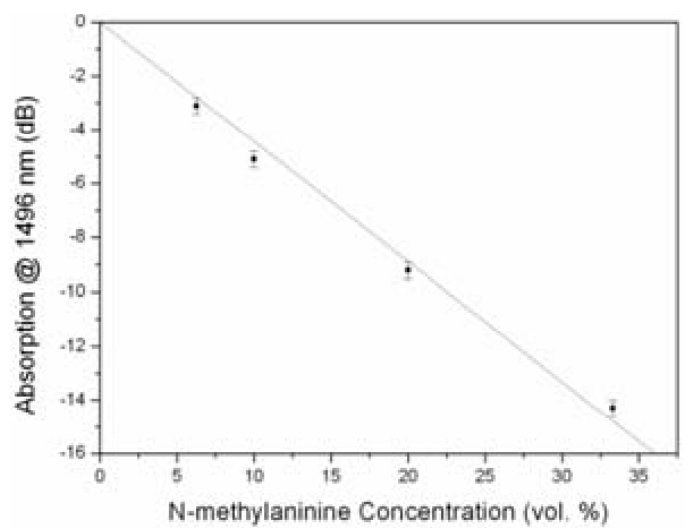

\subsubsection{Testing of the resonator refractometry performance}

For the micro-disk resonator refractometry sensor testing, deionised (DI) water solutions of isopropanol (IPA) of varying concentrations are injected into the PDMS channels through a syringe pump, and the resonant peak shift due to ambient refractive index change is monitored in situ. The measurements with different concentrations of solutions are repeated twice to confirm reproducibility. The TM polarisation transmission spectra of a $\mathrm{Ge}_{17} \mathrm{Sb}_{12} \mathrm{~S}_{71}$ micro-disk resonator in IPA solutions of various concentrations are shown in Figure 6(a). The resonant wavelength shift as a function of IPA concentration and corresponding solution refractive index is plotted in Figure 6(a), and a refractive index (RI) sensitivity of $(182 \pm 5) \mathrm{nm} / \mathrm{RIU}$ is inferred from the fitted curve slope. By applying a Lorentzian fit to the resonant peaks, we show that the resonant wavelength 
can be determined with an accuracy of $\sim 0.1 \mathrm{pm}$ limited by noise [35], corresponding to a refractive index detection limit of $8 \times 10^{-7}$ Refractive Index Unit (RIU). Such a detection limit represents an order of magnitude improvement over commercial surface plasmonic resonance sensors.

Figure 6 (a) TM-polarisation transmission spectra of a Ge-based micro-disk in four IPA solutions of different concentrations and (b) Measured resonant peak wavelength shift as a function of IPA solution molar concentration and corresponding solution refractive index (see online version for colours)

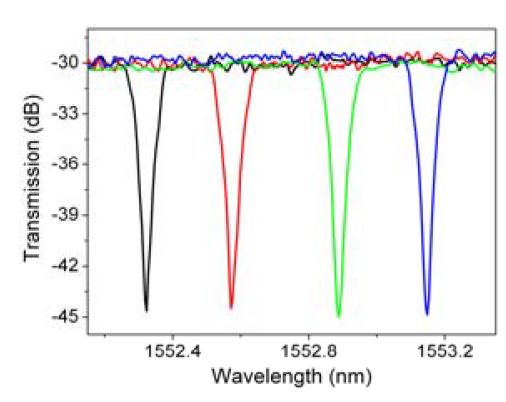

(a)

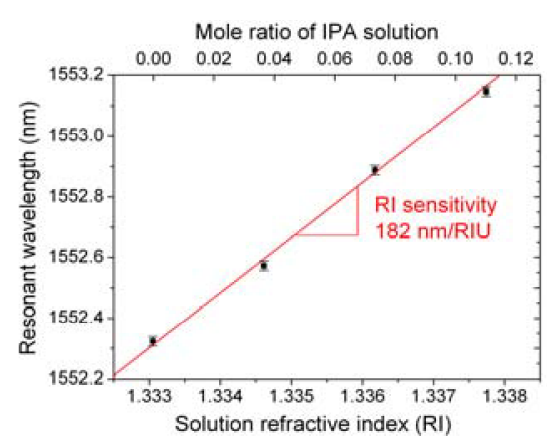

(b)

\section{Conclusions}

An interdisciplinary team of researchers at Clemson University, MIT and University of Central Florida have fabricated and characterised chalcogenide high-index-contrast waveguides with core sizes down to the sub-micron range and micro-resonators with record cavity Q-factors. As a result of our cross-disciplinary research approach blending glass and polymer science, photonic device design and fabrication expertise, along with laser fabrication and characterisation, we have demonstrated a micro-fluidic waveguide evanescent chemical sensor and a micro-fluidic refractive index sensor with an index detection limit of $8 \times 10^{-7}$ RIU. The micro-resonator device is a promising device platform for biochemical sensing and micro-photonics integration.

Advances in materials for the integrated micro-photonic structures discussed here will have a broad impact in the glass materials, sensing and photonics communities. Specifically, we have shown:

- the demonstration of an integrated chalcogenide glass resonator is important for applications that involve strong photon-matter interactions including nonlinear optics and light emission

- the superior resolution to refractive index change enabled by the new chalcogenide resonator device may lead to new measurement paradigms for glass property characterisations and material investigation 
- the novel photonic designs developed such as the pulley-type coupler are applicable to photonic devices in other materials systems

- the lift-off technique can be employed to pattern a variety of devices in a range of oxide and non-oxide glass compositions suitable for diverse infrared sensor applications.

\section{Acknowledgements}

The work presented in this paper is conducted at Clemson University in collaboration with the Laser Plasma Laboratory group at CREOL/UCF and the Microphotonics Center at Massachusetts Institute of Technology. Funding support is provided by the Department Of Energy under award number DE-SC52-06NA27341. The authors also acknowledge the Micro-systems Technology Laboratories at MIT for fabrication facilities.

\section{Disclaimer}

This paper is prepared as an account of work supported by an agency of the US Government. Neither the US Government nor any agency thereof, nor any of their employees, makes any warranty or assumes any legal liability or responsibility for the accuracy, completeness or usefulness of any information, apparatus or process disclosed, or represents that its use would not infringe privately owned rights. Reference herein to any specific commercial product, process, or service by trade name, trademark, manufacturer, or otherwise does not necessarily constitute or imply its endorsement or favouring by the US Government. The opinions of authors expressed herein do not necessarily reflect those of the US Government or any agency thereof.

\section{References}

1 Yager, P., Edwards, T., Fu, E., Helton, K., Nelson, K., Tam, M. and Weigl, B. (2006) 'Micro-fluidic diagnostic technologies for global public health', Nature, Vol. 442, pp.412-418.

2 Moore, G. (1965) 'Cramming more components onto integrated circuits', Electronics, Vol. 38, No. 8, pp.114-117.

3 Hu, J., Tarasov, V., Carlie, N., Petit, L., Agarwal, A., Richardson, K. and Kimerling, L. (2008) 'Exploration of waveguide fabrication from thermally evaporated Ge-Sb-S glass films', Opt. Mater., Vol. 30, pp.1560-1566.

$4 \mathrm{Hu}$, J., Carlie, N., Petit, L., Agarwal, A., Richardson, K. and Kimerling, L. (2008) 'Demonstration of high-Q chalcogenide glass ring resonators', Opt. Lett., Vol. 33, pp.761-763.

$5 \mathrm{Hu}$, J., Carlie, N., Feng, N., Petit, L., Agarwal, A., Richardson, K. and Kimerling, L. (2008) 'Planar waveguide-coupled, high-index-contrast, high-Q resonators in chalcogenide glass for sensing', Opt. Lett., Vol. 33, pp.2500-2502.

$6 \mathrm{Hu}$, J., Feng, N., Carlie, N., Petit, L., Wang, J., Agarwal, A.A., Richardson, K. and Kimerling, L. (2007) 'Demonstration of low-loss high-index-contrast planar glass waveguides with graded-index cladding layers', Opt. Express, Vol. 15, pp.14566. 
$7 \mathrm{Hu}$, J., Tarasov, V., Carlie, N., Feng, N., Petit, L., Agarwal, A., Richardson, K. and Kimerling, L. (2007) 'Si-CMOS-compatible lift-off fabrication of low-loss planar chalcogenide waveguides', Opt. Express, Vol. 15, pp.11798.

8 Hu, J., Tarasov, V., Carlie, N., Feng, N., Petit, L., Agarwal, A., Richardson, K. and Kimerling, L. (2007) 'Fabrication and testing of planar chalcogenide waveguide integrated micro-fluidic sensor', Opt. Express, Vol. 15, pp.2307.

9 Ling, Z., Ling, H. and Cheng Shan, Z. (1995) 'New chalcohalide glasses from the $\mathrm{Sb}_{2} \mathrm{~S}_{3}-\mathrm{MXn}$ system', J. Non-Cryst. Solids, Vol. 184, pp.1-4.

10 Nasu, H., Ibara, Y. and Kubodera, K. (1989) 'Optical third-harmonic generation from some high-index glasses', J. Non-Cryst. Solids, Vol. 110, pp.229-234.

11 Savage, J.A. (1982) 'Optical properties of chalcogenide glasses', J. Non-Cryst. Solids, Vol. 47, pp.101-115.

12 DeCorby, R.G., Ponnampalam, N., Pai, M.M., Nguyen, H.T., Dwivedi, P.K., Clement, T.J., Haugen, C.J., McMullin, J.N. and Kasap, S.O. (2005) 'High index contrast waveguides in chalcogenide glass and polymer', IEEE J. Sel. Top. Quantum Electron., Vol. 11, pp.539-546.

$13 \mathrm{Xu}$, J. and Almeida, R.M. (2000) 'Preparation and characterization of germanium sulfide based sol-gel planar waveguides', J. Sol-Gel Sci. Technol., Vol. 19, pp.243-248.

14 Delaizir, G., Lucas, P., Zhang, X., Ma, H., Bureau, B. and Lucas, J. (2007) 'Infrared glass-ceramics with fine porous surfaces for optical sensor applications', J. Am. Ceram. Soc., Vol. 90, pp.2073-2077.

15 Nakamura, K., Okada, T. and Ueha, S. (2001) 'Measuring the optical path length of a plastic optical fibre using the sing-around method and its sensor applications', J. Opt. A: Pure and Appl. Opt., Vol. 3, pp.L17-L19.

16 Lopez, C. (2004) Evaluation of the Photo-Induced Structural Mechanisms in Chalcogenide Glass, PhD Thesis, College of Optics and Photonics at the University of Central Florida.

17 Petit, L., Carlie, N., Richardson, K., Guo, Y., Schulte, A., Campbell, B., Ferreira, B. and Martin, S. (2005) 'Effect of the substitution of S for Se on the structure of the glasses in the system $\mathrm{Ge}_{0.23} \mathrm{Sb}_{0.07} \mathrm{~S}_{0.70-\mathrm{x}} \mathrm{Se}_{\mathrm{x}}$ ', J. Phys. Chem. Solids, Vol. 66, pp.1788-1794.

18 Viens, J-F., Meneghini, C., Villeneuve, A., Galstian, T.V., Knystaunas, E.J., Duguay, M.A., Richardson, K.A. and Cardinal, T. (1999) 'Fabrication and characterization of integrated optical waveguides in Sulfide Chalcogenide glasses', J. Lightwave Technol., Vol. 17, pp.1184-1190.

19 Luther-Davies, B., Rode, A.V., Madsen, N. and Gamaly, E.G. (2005) 'Picosecond high-repetition-rate pulsed laser ablation of dielectrics: the effect of energy accumulation between pulses', Opt. Eng., Vol. 44, pp.051102.

20 Carlie, N., Hu, J., Petit, L., Agarwal, A., Kimerling, L.C. and Richardson, K. (2008) 'Comparison of the optical and thermal properties and structure of Ge-Sb-S thin films deposited using thermal evaporation and pulsed laser deposition techniques', submitted to Thin Solid Films.

21 Zoubir, A. (2004) Towards Direct Writing of 3-D Photonic Circuits using Ultrafast Lasers, $\mathrm{PhD}$ Thesis, College of Optics and Photonics at the University of Central Florida.

22 Aitken, B.G. and Ponader, C.W. (1999) 'Physical properties and Raman spectroscopy of GeAs sulphide glasses', J. Non-Cryst. Solids, Vols. 256, 257, pp.143-148.

23 Anderson, T., Petit, L., Carlie, N., Choi, J., Hu, J., Agarwal, A.M., Kimerling, L.C., Richardson, K. and Richardson, M. (2008) 'Femtosecond laser photo-response of $\mathrm{Ge}_{23} \mathrm{Sb}_{7} \mathrm{~S}_{70}$ films', Opt. Express, Vol. 16, No. 24, pp.20081-20098.

24 Arsova, D., Skordeva, E., Pamukchieva, V. and Vateva, E. (2005) 'Photoinduced changes in Ge-As-S thin films with various network rigidities', J. Optoelectron. Adv. M., Vol. 7, pp.1259-1264. 
25 Marquez, E., Bernal-Oliva, A.M., Gonzalez-Leal, J.M., Prieto-Alcon, R. and Wagner, T. (2006) 'Optical properties and structure of amorphous $\left(\mathrm{As}_{0.33} \mathrm{~S}_{0.67}\right)_{100-\mathrm{x}} \mathrm{Te}_{\mathrm{x}}$ and $\mathrm{Ge}_{\mathrm{x}} \mathrm{Sb}_{40-\mathrm{x}} \mathrm{S}_{60}$ chalcogenide semiconducting alloy films deposited by vacuum thermal evaporation', J. Phys. D: App. Phys., Vol. 39, pp.1793-1799.

26 Yamashita, M., Yamanaka, H. and Wakabayashi, H. (1996) 'Thin-film preparation of the $\mathrm{Li}_{2} \mathrm{~S}-\mathrm{GeS}_{2}-\mathrm{Ga}_{2} \mathrm{~S}_{3}$ glass system by sputtering', Solid State Ionics, Vol. 89, pp.299-304.

27 Zakery, A., Ruan, Y., Rode, A.V., Samoc, M. and Luther-Davies, B. (2003) 'Low-loss waveguides in ultrafast laser-deposited $\mathrm{As}_{2} \mathrm{~S}_{3}$ chalcogenide films', J. Opt. Sci. Am. B, Vol. 20, pp.1844-1852.

28 Nazabal, V., Nemec, P., Jedelsky, J., Duverger, C., Le Person, J. and Adam, J.L. (2004) 'Dysprosium-doped chalcogenide films prepared by pulsed-laser deposition', Proc. SPIE, Vol. 5451, pp.543-549.

29 Rode, A.V., Zakery, A., Samoc, M., Charters, R.B., Gamaly, E.G. and Luther-Davies, B. (2002) 'Laser-deposited $\mathrm{As}_{2} \mathrm{~S}_{3}$ chalcogenide films for waveguide applications', Appl. Phys. Sci., Vols.197-198, pp.481-485.

30 Miyauchi, K., Qiu, J., Shojiya, M., Kawamoto, Y. and Kitamura, N. (2001) 'Structural study of $\mathrm{GeS}_{2}$ glasses permanently densified under high pressures up to $9 \mathrm{GPa}$ ', J. Non-Cryst. Solids, Vol. 279, pp.186-195.

31 Nemec, P., Jedelsky, J., Frumar, M., Munzar, M., Jelinek, M. and Lancok, J. (2003) 'On the optical properties of amorphous Ge-Ga-Se films prepared by pulsed laser deposition', J. Non-Cryst. Solids, Vols. 326, 327, pp.53-57.

32 Madden, S., Choi, D.Y., Bulla, D.A., Rode, A.V., Luther-Davies, B., Ta'eed, V.G., Pelusi, M.D. and Eggleton, B.J. (2007) 'Long, low loss etched As2S3 chalcogenide waveguides for all-optical signal regeneration', Opt. Express, Vol. 15, No. 22, pp.14414-14421.

33 Choi, D.Y., Madden, S., Rode, A., Wang R. and Luther-Davies, B. (2007) 'Fabrication of low loss $\mathrm{Ge}_{33} \mathrm{As}_{12} \mathrm{Se}_{55}$ (AMTIR-1) planar waveguide', Appl. Phys. Lett., Vol. 91, pp.011115.1-011115.3.

34 Shaji, S., Eappen, S., Rasheed, T. and Nair, K. (2004) 'NIR vibrational overtone spectra of N-methylaniline, N,N-dimethylaniline and N,N-diethylaniline - a conformational structural analysis using local mode model', Spectrochim. Acta, Part A, Vol. 60, pp.351-355.

$35 \mathrm{Hu}$, J., Sun, X., Agarwal, A. and Kimerling, L. (2009) 'Detection limit of optical resonator biochemical sensors', J. Opt. Soc. Am. B, Vol. 26, No. 5, pp.1032-1041. 